"You know, when you suspect something, it's always better when it turns out to be true »: Mémoire et média dans l'épisode « The Entire History of You » (S01E03) de Black Mirror (2011-)

\title{
David Roche
}

\section{OpenEdition}

Journals

Édition électronique

URL : http://journals.openedition.org/tvseries/3094

DOI : $10.4000 /$ tvseries.3094

ISSN : 2266-0909

Éditeur

GRIC - Groupe de recherche Identités et Cultures

Référence électronique

David Roche, « "You know, when you suspect something, it's always better when it turns out to be true » : Mémoire et média dans l'épisode « The Entire History of You » (S01E03) de Black Mirror (2011-) », TV/Series [En ligne], 14 | 2018, mis en ligne le 31 décembre 2018, consulté le 21 avril 2019. URL : http://journals.openedition.org/tvseries/3094; DOI : 10.4000/tvseries.3094

Ce document a été généré automatiquement le 21 avril 2019.

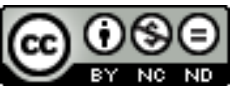

TV/Series est mis à disposition selon les termes de la licence Creative Commons Attribution - Pas d'Utilisation Commerciale - Pas de Modification 4.0 International. 
"You know, when you suspect something, it's always better when it turns out to be true »: Mémoire et média dans l'épisode « The Entire History of You » (S01E03) de Black Mirror (2011-)

\author{
David Roche
}

1 "You know, when you suspect something, it's always better when it turns out to be true » : c'est ce que dit Liam, le protagoniste de «The Entire History of You » (S01E03 de Black Mirror), à son amie Ffion, dont il vient d'apprendre qu'il y a dix-huit mois, cinq jours après qu'il l'a quittée, elle a couché avec un ancien amant nommé Jonas. Il me semble que cette remarque fait écho à plusieurs niveaux à celle émise par Fred Madison dans Lost Highway (1997) de David Lynch: "I like to remember things my own way. How I remembered them. Not necessarily the way they happened ». Et ce de façon contrastive : elle est prononcée à la fin de l'épisode (et non pas au début du film), par la victime de la trahison (et non pas par le meurtrier potentiel), il est question de soupçon (et non pas de souvenir) corroboré par un implant numérique (et non pas par une bande magnétique). Et pourtant, les deux remarques laissent entrevoir une même perception de la technologie : elle a valeur de preuve dans un acte transgressif (effraction, trahison) et est intimement liée à la notion de vérité. Autrement dit, son ontologie est perçue comme photographique. Ce qui change n'est donc pas tant la perception que le sujet a de l'ontologie du médium, mais l'emploi qu'il ou elle en fait et la valeur qu'il ou elle lui attribue : Fred rejette toute prothèse mnésique ; Liam, lui, adhère à une technologie qui, comme le titre de l'épisode l'indique, permet de stocker l'histoire audiovisuelle de sa vie.

Cette technologie, le Grain, est un implant relié au système perceptivo-cognitif : l'œil est transformé en caméra et apparemment en écran, tandis que les images sont 
vraisemblablement archivées dans l'implant. Cette prothèse mnésique fonctionne en parallèle du cerveau, et donc de la mémoire humaine. D'un point de vue purement technique, le Grain transforme le sujet non pas en cyborg mais en humain augmenté. Le sujet augmenté du Grain correspondrait, dans la typologie de Thierry Hoquet $^{1}$, à l'Organorg - un humain qui peut se passer de son équipement (comme Batman) - plutôt qu'au Cyborg qui n'est rien sans la technologie (comme Robocop). Et pourtant «The Entire History of You » (tout comme les épisodes S03E01 et S03E05) laisse entendre qu'en pratique, la distinction n'est pas si claire. L'utilisation du Grain situerait le sujet dans un entre-deux-Cyborg-et-Organorg pour plusieurs raisons: d'abord, l'installation et l'extraction de l'implant nécessitent une opération, et surtout l'épisode révèle que l'humain augmenté en vient à perdre " une partie de son autonomie », soit qu'il ou elle perde certaines facultés (la mémoire) ou, pire, subisse le contrôle du pouvoir. L'épisode « Nosedive » (S03E01), dans lequel les personnages sont dotés d'un implant similaire (cette fois-ci, un réseau social leur permettant de noter les autres lors d'interactions), arrive aussi à la conclusion que la frontière entre Cyborg et Organorg, c'est-à-dire entre dépendance et autonomie, est bien ténue, tandis que les épisodes «Playtest» (S03E02) et «Men Against Fire » (S03E05) jouent sur l'inquiétude déclenchée par le fait qu'un implant fasse de l'humain un cyborg que l'on peut contrôler. Dans l'épisode «Black Museum » (S04E06), l'introduction d'une conscience dans un corps opéré par une interface relève clairement du Cyborg.

3 La relation entre mémoire et humanité est au cœur de bon nombre de fictions imaginant le posthumain. Dans l'une des plus célèbres au cinéma, Blade Runner (Ridley Scott, 1982), les Réplicants pensent qu'ils sont humains parce qu'ils ont été dotés d'une mémoire artificielle, et les débusquer nécessite de tester leur empathie. Et pourtant, la fin du premier volet montre en quoi leur point de vue est justifié à travers le monologue « Tears in Rain ", dans lequel Roy Batty évoque des événements qui l'ont ému et dont le souvenir le touche: "I've seen things you people wouldn't believe. Attack ships on fire off the shoulder of Orion. I watched C-beams glitter in the dark near the Tannhäuser Gate. All those moments will be lost in time, like tears in rain ». L'humain n'est pas qu'un être doté d'une mémoire: il est pourvu d'une sensibilité, notamment esthétique, qui peut être provoquée par l'artifice. Dans Blade Runner 2049 (Denis Villeneuve, 2017), la docteure Ana Stelline le confirme quand elle répond à l'agent $\mathrm{K}$ que l'on sait qu'un souvenir est réel parce qu'on le ressent comme tel.

4 La mémoire du posthumain, l'être et la mémoire, mémoire et sensible : ce n'est pas la nouveauté des thèmes qui caractérise Black Mirror, série qui s'inscrit dans un héritage SF cinématographique, littéraire et télévisuel (dont la filiation à The Twilight Zone) tout à fait assumé. D'ailleurs, si Charlie Brooker explique le nom de la série comme renvoyant à notre société de l'écran ("The "black Mirror" of the title is the one you'll find on every wall, on every desk, in the palm of every hand: the cold, shiny screen of a TV, a monitor, a smartphone $\left.{ }^{2} »\right)$, ce titre empreinte directement au roman auquel on attribue l'invention du terme "cyberpunk", Neuromancer (1984) de William Gibson, dans lequel figurent également des implants ( And on the far rim of consciousness, a scurrying, fleeting impression of something rushing toward him, across leagues of black mirror $\left.{ }^{3} »\right)$. Je voudrais cependant insister dans cet article sur le fait que, dans Black Mirror, l'exploration de la technologie dépasse largement l'allégorie ; elle se situe également, à divers degrés selon les épisodes, au niveau de sous-textes qui proposent des discours sur les médias, et donc sur des enjeux sociétaux actuels, comme l'a montré Sébastien Lefait au sujet de 
«Men Against Fire 4 ». En ce sens, la série opère de la même manière que le splendide Her (Spike Jonze, 2013) dans l'analyse approfondie de Donna Kornhaber : de la réflexion sur l'identité (genrée, humaine, etc.) qu'encourage le posthumain en découle une autre sur le devenir du médium (ou plutôt des médias ${ }^{5}$ ). Dans Black Mirror, plus précisément, il s'agit de décliner à chaque épisode une réflexion sur le rôle de la spectatrice/du spectateur et sur le récit audiovisuel à l'ère du numérique et de la culture de la convergence décrite par Henry Jenkins ${ }^{6}$. Autrement dit, il s'agit d'une réflexion sur les médias mêmes qui permettent la circulation de la série.

\section{Potentiels utopiques et dystopiques}

Comme l'immense majorité des œuvres de science-fiction au cinéma et à la télévision ${ }^{7}$, la série Black Mirror est dans l'ensemble largement technophobe ou plus précisément critique de l'emploi qu'on fait des technologies ${ }^{8}$. Le seul épisode des trois premières saisons qui fasse la part belle à l'utopie (et ce malgré un épilogue ambigu ${ }^{9}$ ) est le touchant "San Junipero » (S03E04), dans lequel la réalité virtuelle permet non seulement aux sujets en phase terminale de cancer d'oublier leur souffrance physique en vivant une vie alternative dans l'univers de leur choix, mais aussi aux deux protagonistes (Kelly et Yorkie) de vivre une relation amoureuse interraciale, malgré leur vie passée dans une hétérosexualité imposée. La saison 4 poursuit la veine utopique dans les épisodes «USS Callister » (S04E01) où des clones virtuels s'affranchissent de leur créateur, et « Hang the DJ » (S04E04) où l'amour est synonyme de rébellion contre un système. Les épisodes « San Junipero » et " Hang the DJ", qui se font écho de par leur position dans leurs saisons respectives, rejoignent ainsi le club très fermé des fictions audiovisuelles - Blade Runner (1982) et Blade Runner 2049 (2017), bien sûr, mais aussi Battlestar Galactica (Scifi, 2003-2008) et $\mathrm{Her}$ (2013) - qui ne limitent pas la question du posthumain à une relation de domination-soumission mais la pensent plutôt par le prisme plus positif de l'amour comme rencontre avec autrui.

6 «The Entire History of You » n'échappe pas à la dominante technophobe. L'accent est mis sur le potentiel dystopique de la technologie: surveillance et contrôle, caractère obsédant, relation problématique entre réel et virtuel. Dès la troisième scène, il apparaît clairement que le Grain sert une société de sousveillance ${ }^{10}$ où les dispositifs opèrent au niveau de l'humain, quand un agent de la sécurité demande à Liam de bien vouloir le laisser scanner les images de ses dernières 48 heures, même si nous ignorons si ces données sont seulement passées au crible ou seront également stockées (cf. Fig. 1). 


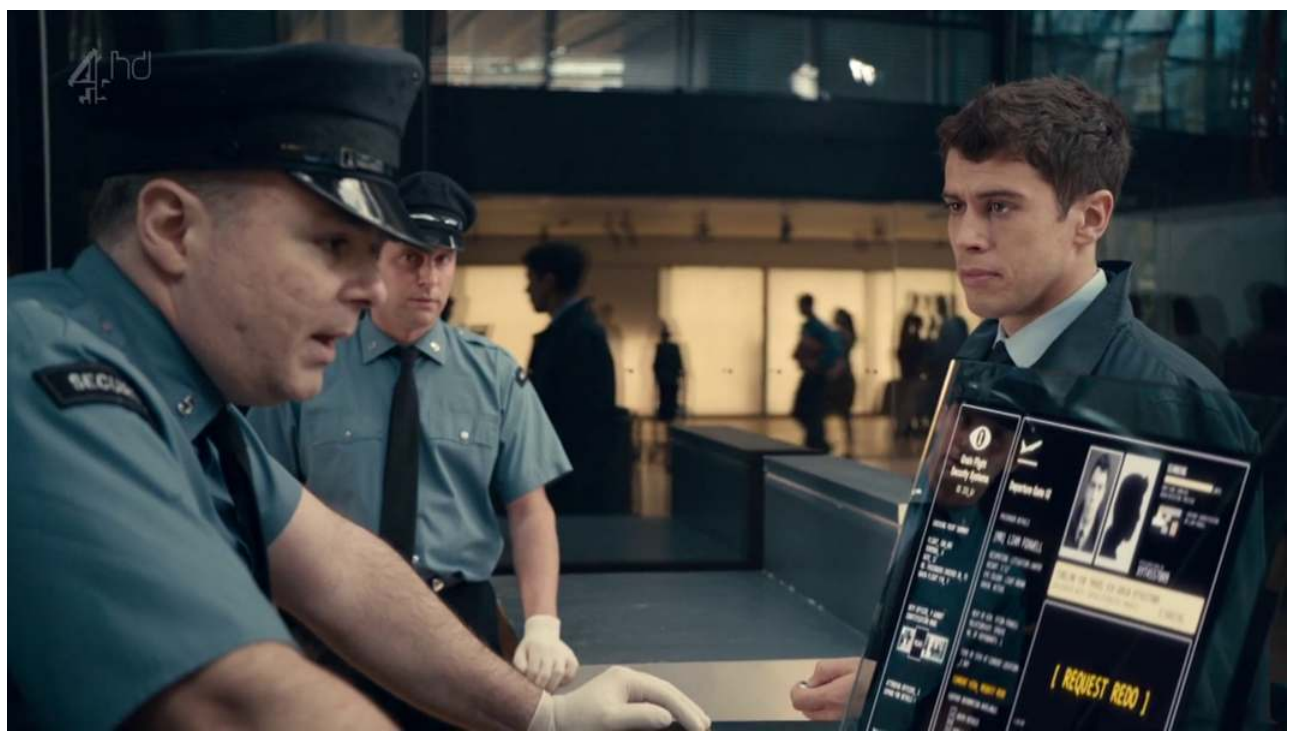

Fig 1 : Les données de Liam sont scannées

7 Dès la deuxième scène, Liam se repasse le film de son entretien, et le temps qu'il passe à revisiter ses souvenirs archivés ne fait que s'accroître tout au long de l'épisode (45s dans l'acte 1 , plus de 2 minutes dans l'acte 2, plus de 8 minutes dans l'acte 3 et environ 5 minutes dans l'acte $\left.4^{11}\right)$. Dans la scène où ils font l'amour, Liam et $\mathrm{Ffi}$ se stimulent en se repassant des images d'actes passés (cf. Fig. 2).

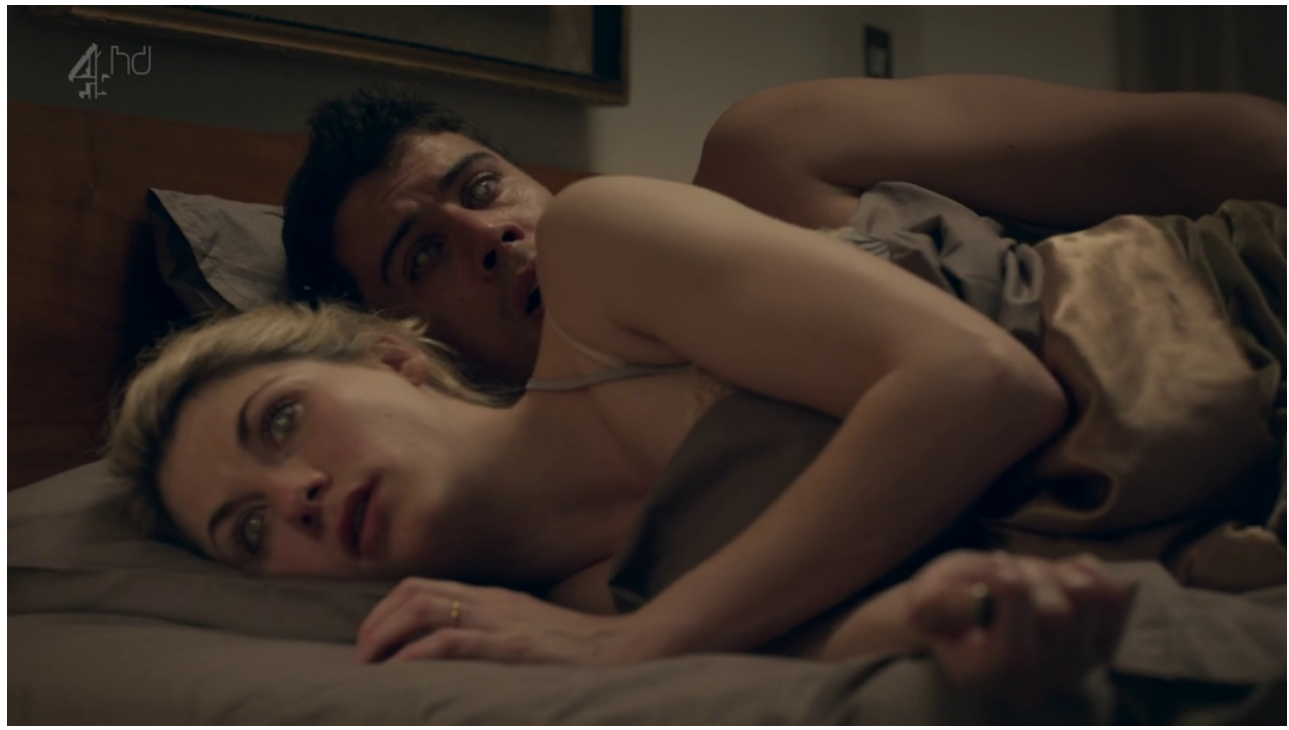

Fig 2 : Liam et Ffi se repassent des images d'actes passés

8 L'analyse du comportement passé de Ffi conduit Liam à ignorer la manière dont elle le considère dans le présent, comme Ffi le lui fait remarquer : « And how am I looking at you now, Liam? ». Et dans les dernières scènes, Liam voit encore Ffi et leur fille Jodie dans la maison pourtant vide. Il n'est pas sous-entendu que Liam confond les deux niveaux ontologiques, mais plutôt que le virtuel pallie clairement un manque dans le réel, et que l'immersion dans le virtuel induit une sorte de stupeur passive ${ }^{12}$.

9 Même l'intérêt principal du Grain est remis en question dans une brève scène apparemment anodine. Quand Liam arrive chez Lucy et Paul, il s'arrête un moment pour se repasser un souvenir. On comprend rétrospectivement que ce qu'il cherchait, c'était le 
nom de l'hôtesse de cette soirée. Cette scène semble montrer que la technologie pallie les déficiences humaines, mais sachant que la mémoire se travaille et ce depuis l'enfance (l'enfant de Ffi et Liam s'avère d'ailleurs être déjà équipé, comme la fille de Marie dans l'épisode «Arkangel»), on comprend que le Grain présente le risque de mener à la nécrose de la mémoire humaine. Cela rendrait presque crédible l'excuse de Liam lorsqu'il change d'avis après avoir invité Jonas à boire un verre chez eux : «No, it's just that Actually, it turns out we're tired. And, uh, I guess we forgot, but, now we've remembered, so... Sorry. »

10 Le potentiel utopique du Grain est pourtant indéniable: possibilité d'enregistrer, d'archiver ou d'éliminer des scènes audiovisuelles du passé ; possibilité de partager ces scènes, d'en discuter, de les analyser. Quand Jeff et Lucy apprennent que Liam a subi une réunion d'évaluation au travail, ils lui proposent de visionner son entretien ensemble afin de lui faire part de leurs impressions et lui faire un retour (sous forme de feedback). La proposition articule parfaitement les trois concepts clés de la culture de la convergence médiatique selon Jenkins : transférabilité de ces scènes vers une multitude d'interfaces, « intelligence collective » et «culture participative » à travers le partage de savoirs et de savoir-faire dans le travail de groupe. Pourtant, la proposition est immédiatement remise en question pour des raisons éthiques. L'intervention de Jonas souligne que le collectif est une force de pression plutôt que de soutien (Liam subit une forme de "peer-pressure»), les attitudes de Lucy et de Jeff trahissant à la fois la légèreté ( « Well, it could be fun, we could, hmm - vote! ») et la condescendance («I work in recruitment, actually, so it might be quite useful for you - going forward »), avec laquelle ils envisagent la possibilité que Liam perde son emploi (il vient de se présenter comme suit: " soon to be unemployed/ unemployable lawyer »). La scène est avant tout reçue comme un spectacle consommable, à l'image d'une émission de téléréalité (« Liam’s going to redo his appraisal so we can give him marks!») plutôt que comme une situation compliquée vécue par l'ami d'une amie de longue date ${ }^{13}$.

11 C'est que la soirée tout entière est centrée sur les spectacles qui figurent à l'écran : la scène que diffuse Jeff avant l'arrivée de Liam, ou la scène dans la boîte de nuit qui clôt la soirée (cf. Fig. 3) :

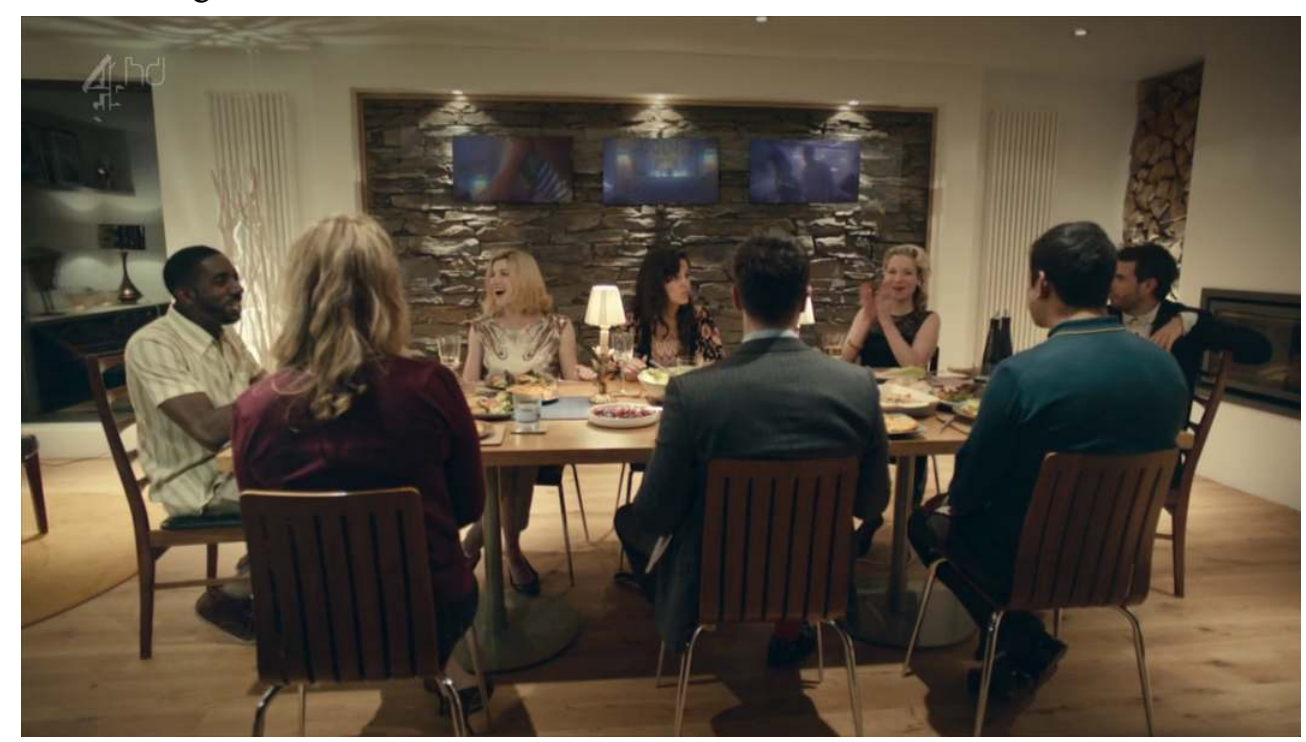

Fig. 3 : Scène dans la boîte de nuit 
Ceux-ci servent de cadre à la structure du premier acte de l'épisode. La conversation à table elle-même ramenée en revient au Grain : la preuve ultime selon Jonas que l'amour monogame éternel est un idéal factice réside dans le fait que lui-même se repasse régulièrement les images de ses ébats sexuels passés. L'interface technologique parasite les relations humaines, bien qu'elle ne soit vraisemblablement pas la cause de ses maux. La critique de ce parasitage est incarnée au niveau diégétique par le personnage de Halem qui, de manière significative, arrive en cours de soirée. La narration lui laisse d'ailleurs le dernier mot lors du débat pro- ou anti-Grain ${ }^{14}$. Halem a perdu son Grain non pas par conviction politique (on apprend qu'il existe un mouvement anti-Grain que l'on devine être un mouvement de résistance qui s'élève notamment contre la société de sousveillance), mais parce qu'un ancien amant le lui a arraché ; elle se trouve néanmoins plus heureuse en allant à contre-courant de la norme (en anglais, " against the grain »). Jonas demande alors à toucher sa cicatrice. Les convives sont visiblement gênés, mais la curiosité de Jonas donne lieu à un échange tactile, sensuel, entre lui et Halem («Well, it feels quite nice ») qui conteste ainsi la toute-puissance du regard - les autres convives détournent (pour la plupart) le leur. Le fait que Jonas passe la nuit avec Halem plutôt qu'avec l'autre célibataire de la soirée, la pro-Grain Colleen, donne une suite à cette interaction physique qui contraste avec la scène d'amour entre Liam et de Ffi, qui se nourrissait des scènes du passé. Le discours technophobe de l'épisode valorise ainsi le sens (le toucher) impliquant un contact plus direct entre sujets plutôt que les sens (le regard, l'ouïe) permettant une plus grande distance et une médiation écranique ${ }^{15}$.

\section{Acteur-spectateur-critique}

Doté d'un implant équivalent à une caméra qui enregistre en continu, le sujet devient un acteur ou même une star, dans son film comme dans ceux des autres. Le monde entier devient une scène - ou plutôt un plateau ou un lieu de tournage. En début de soirée, Jeff projette une «rediffusion» ("redo») et se plaint à Ffi et à Jonas d'une chambre d'hôtel qu'il a payée à prix d'or : «I'm not being funny, right, but look at that. [...] That. Frayed carpet. There, you see. [...] This is a five-star suite. I paid good money to have perfect details. Now I've got that shitty carpet for the rest of my life » (cf. Fig. 4).

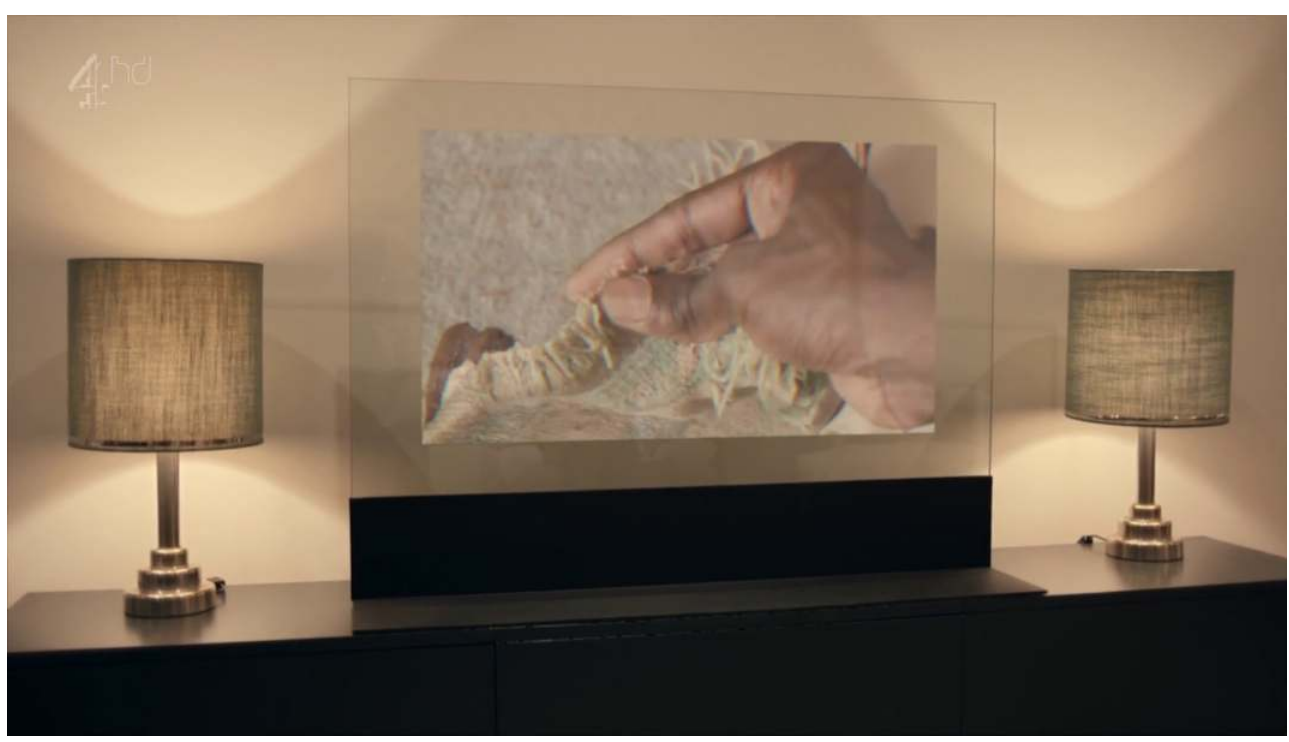


14 Le souvenir a été gâché non pas par un événement, mais par un détail dans la composition qui aurait pu être relayé aux oubliettes de la mémoire humaine : grâce à la technologie (Jeff effectue un grossissement de l'image incriminante), l'information devient un surplus qui parasite l'essentiel (nous ignorons d'ailleurs ce que Jeff faisait dans cette chambre: où était-ce? quand? était-il seul, en vacances ou en déplacement professionnel ?). La mémoire artificielle se distingue donc de la mémoire humaine (moins précise, plus sélective et plus subjective) par sa capacité à mettre toutes les informations au même niveau, idée qui est visualisée par l'image du cercle de données. Le Grain construit un sujet conscient que tout sera enregistré, et donc «self-conscious » dans les différents sens du termes : conscient de soi, certes, mais aussi mal dans sa peau. «I'm just happier now ", avoue Halem, maintenant qu'elle n'a plus de Grain. Un sujet peut tenter de réguler et réviser son jeu, comme le fait Liam lors de l'évaluation où il répond à un mot d'esprit de Max, d'abord par un «Yes » assez réservé, en hochant de la tête, puis avec le sourire, un mouvement plus large et avenant et un ton plus enjoué, "Yes, I see that ". Lors de la soirée, la plupart des personnages limitent leurs interventions afin d'éviter de se livrer, tandis que d'autres assument leur statut de star: Lucie, l'hôtesse de soirée ; et surtout Jonas, le "Mister Cool», au centre des regards et placé en tête de table, naturellement ${ }^{16}$.

15 Le Grain fait du sujet un acteur, mais c'est surtout sur le sujet en tant que spectateur que "The Entire History of You » insiste ${ }^{17}$, et ce selon deux modalités. Tout d'abord, en nous donnant à voir, selon une narration classique, le visage du protagoniste dans des contrechamps dont les angles varient (frontaux, de profil). L'entretien qui sert de prologue à l'épisode présente Liam (en plan rapproché) comme un personnage qui observe et réagit aux autres. La fonction de spectateur est renforcée par l'effet secondaire induit par le Grain quand le sujet se repasse les enregistrements : son iris devient blanc, signifiant que le visionnage est aussi un aveuglement à son environnement réel.

En deuxième lieu, c'est le dispositif même du Grain qui construit le sujet comme regard. Équivalent à des plans-séquences en caméra subjective, les "rediffusions » («redoes») nient la possibilité du contre-champ. Le sujet est donc relégué à l'en-deçà qu'il ne peut franchir que de façon très limitée (apparition des mains), sauf à avoir recours à une surface réfléchissante (comme le miroir de la salle de bain dans la dernière scène). Autrement dit, si l'usage que font les personnages du Grain rappelle bien évidemment l'emploi contemporain des smartphones et avant cela des home videos avec caméra à l'épaule ou à la main, l'implant ne permet pas le contre-champ et la prise de soi (et donc l'équivalent de selfies), sauf à effectuer un montage à partir des scènes prises par un autre sujet. Sujet sans cesse conscient du regard, donc, mais sans regard sur soi, puisque le Grain ne le permet pas du fait qu'il est intégré au corps. D'où la ligne ténue entre Organorg et Cyborg discutée en introduction, et l'opposition entre toucher et vision mentionnée en amont (au niveau sonore, c'est un peu différent parce que le Grain enregistre parfaitement le sujet doté de l'implant). Les rediffusions ressemblent finalement plus aux expérimentations hollywoodiennes en caméra subjective de 1947 comme La Dame du lac de Robert Montgomery et Les Passagers de la nuit de Delmer Daves, et posent les mêmes problèmes au niveau spectatoriel ${ }^{18}$, car ce n'est pas au sujet du souvenir que le spectateur s'identifie mais à l'objet du souvenir. Memory becomes memorabilia, dirait-on en anglais. Le Grain ne propose pas l'expression sensible d'un 
événement passé, mais livre au regard les expressions passées des co-acteurs de cet événement. Il induit donc simultanément une réduction de la subjectivité humaine à l'œil de la caméra et une aliénation du sujet-spectateur vis-à-vis de son soi passé principalement réduit à l'état d'œil-caméra ; lors du rapport sexuel, par exemple, le sujet n'a pas accès à l'image de son plaisiri ${ }^{19}$.

Par ailleurs, la représentation de la consommation d'images dans cet épisode est vraisemblablement influencée par les deux paradigmes théoriques selon lesquels elle a été pensée par les théoriciens du cinéma: le modèle psychanalytique sur lequel s'appuient Jean-Louis Baudry, Christian Metz et Laura Mulvey ${ }^{20}$ quand ils conçoivent le spectateur de cinéma comme un voyeur passif, immergé dans le spectacle et assujetti à l'idéologie et à des processus inconscients; le paradigme cognitif sur lequel s'appuient David Bordwell, Murray Smith et Ed $\operatorname{Tan}^{21}$ quand ils postulent que le spectateur tient un rôle actif dans la construction du récit et réagit au film selon des schèmes cognitifs ${ }^{22}$. Les exemples d'expérience purement immersive sont peu nombreux dans «The Entire History of You » : la première a lieu quand Ffi et Liam font l'amour; la seconde quand Liam, couché ou errant dans la maison, se remémore leur vie passée. Dans les deux cas, on retrouve des aspects renvoyant au paradigme psychanalytique: voyeurisme, passivité, caractère obsessionnel, régression, tentative de combler un manque (sexuel, affectif). Là encore, la lumière blanche qui illumine l'iris renvoie à l'éblouissement du spectateur face au spectacle ${ }^{23}$.

18 La seconde modalité de consommation d'images est bien plus fréquente. Liam passe son temps à revoir les événements passés pour les analyser. Il interagit avec ses rediffusions selon le schéma classique perception-cognition-action, c'est-à-dire perception du stimulus, traitement des données, action en conséquence. C'est ce qui justifie vraisemblablement l'emploi de la télécommande, alors que l'on aurait pu penser qu'une technologie aussi sophistiquée soit intégrée à l'implant ${ }^{24}$. En effet, celle-ci permet de dramatiser l'action du sujet-spectateur, effet renforcé par le montage qui alterne entre images du présent et du passé, d'une part, et plans du visage de Liam et de son pouce glissant sur la télécommande de l'autre. Finalement, ce sont les raccords qui symbolisent les processus cognitifs.

19 Dans «The Entire History of You » et "Men Against Fire », les sujets dotés d'implants sont donc des «revoyants", comme le souligne Sébastien Lefait ${ }^{25}$. Permettre au sujet d'être le spectateur de sa vie, c'est in fine lui permettre de l'analyser, et donc d'être le critique de l'histoire audiovisuelle de sa vie. Le Grain facilite ce travail puisqu'il permet de grossir ou de faire un arrêt sur image et de repasser des scènes en boucle. Liam identifie des détails, émet des hypothèses. Dans le taxi, suite à son entretien, il repère le lapsus de Max et le transforme en détail saillant. La répétition de cette scène a un caractère obsédant, mais elle permet également de mettre en avant qu'un même film peut donner lieu à différentes interprétations. Si Liam s'est arrêté sur le lapsus de Max, Ffi, elle, remarque que Leah, pendant l'interview, semble avoir coché une case, geste qu'elle interprète comme potentiellement favorable à Liam. La réaction de Ffi indique que la focalisation sur le lapsus dans l'interprétation de Liam se faisait forcément au détriment d'autres signes et d'autres interprétations.

Le sujet fait donc un travail d'interprétation similaire à celui du critique, du cinéphile ou du fan. Si le Grain menace la mémoire humaine, il implique néanmoins un développement potentiel des capacités d'analyse du sujet et il lui permet d'effectuer cette activité collectivement, tout comme les nouvelles technologies à l'ère de la culture de la 
convergence. Néanmoins, l'épisode insiste lourdement sur les limites de cette technologie et de son usage. Premièrement, rien dans "The Entire History of You » ne laisse penser que les usagers transfèrent leurs capacités d'analyse à un questionnement du régime dystopique ${ }^{26}$ (au contraire, il est clairement indiqué que la résistance provient de ceux qui ont abandonné le Grain); cette limite fait écho aux regrets émis par Jenkins concernant l'utilisation des savoir-faire par les communautés de fans, qui se limitent au domaine ludique plutôt que politique ${ }^{27}$. En deuxième lieu, les rediffusions ne sont justement pas des scènes de fiction déterminées par des conventions narratives et génériques, et leurs contextes d'interprétation font souvent défaut. Cette limite réduit par conséquent l'efficacité de la sousveillance ${ }^{28}$. En fait, si le dispositif permet d'analyser autrui, il ne permet pas l'auto-analyse ne serait-ce que comportementale du fait de l'absence de champ/contre-champ; dès lors, on imagine mal en quoi Jeff aurait pu conseiller Liam sans voir ses réactions lors de l'entretien initial. C'est d'ailleurs certainement cette caractéristique du dispositif qui permet aux convives de déshumaniser l'expérience du fait de la dimension aliénante de la caméra subjective : ils n'ont pas à s'embarrasser des expressions faciales d'un sujet réduit à un œil-caméra.

\section{Nouvelles technologies, anciennes problématiques}

L'usage que fait Liam du Grain dans son enquête concernant la relation entre Ffi et Jonas repose sur la certitude que les rediffusions contiennent la vérité. À cette technologie est donc attribuée la même caractéristique ontologique qu'à l'image photographique: l'« indicialitée ${ }^{29}$ ", terme employé par Rosalind Krauss, dont les précurseurs comprennent «l'objectivité essentielle ${ }^{30}$ » d'André Bazin, la «trace ${ }^{31}$ » de Susan Sontag ou encore le " ça-a-étés ${ }^{32}$ " de Roland Barthes ${ }^{33}$. Les images sur lesquelles se concentrent Liam ont valeur de preuve. Y figurent autant d'indices:le lapsus de Max l'intervieweur; le comportement de Ffi à table; le baiser de Ffi et de Jonas en arrière plan de Lucie et Paul qui s'amusent dans la boîte de nuit; et enfin, le tableau sur le mur de la chambre de Ffi et Liam que ce dernier repère dans un souvenir de Jonas (cf. Fig. 5) :

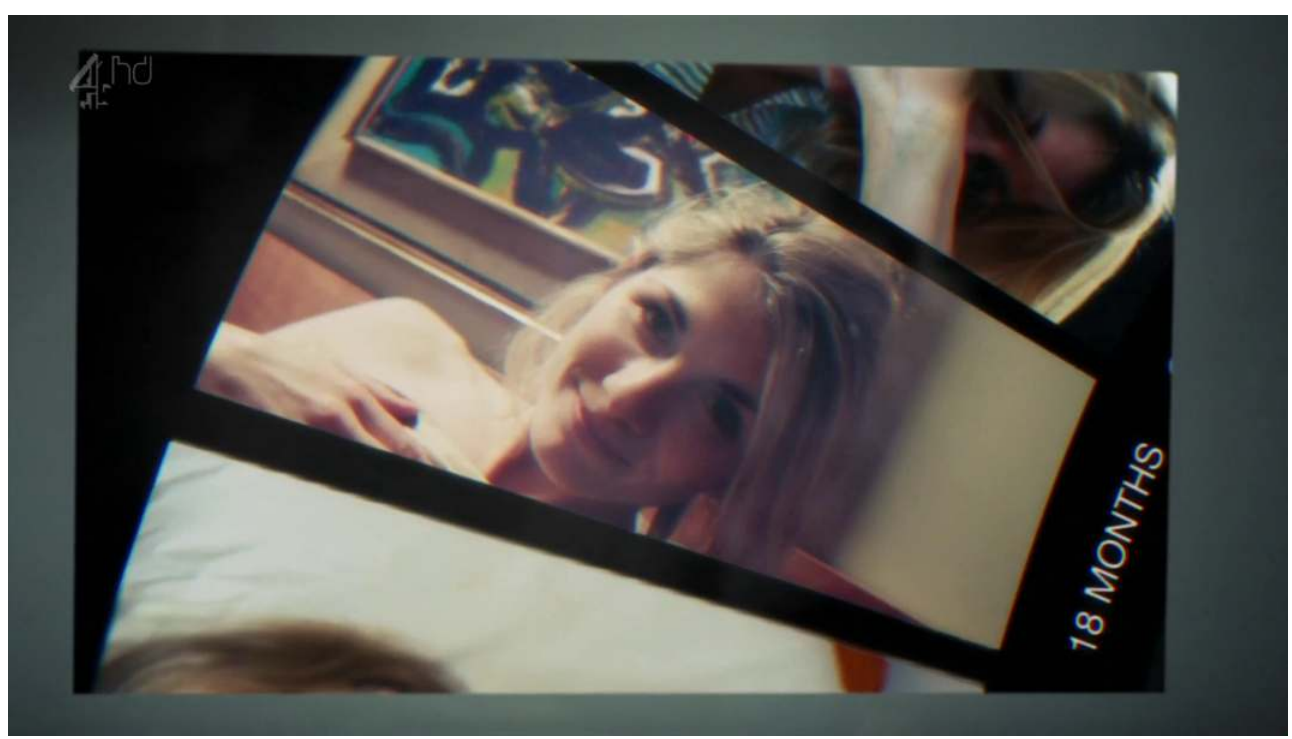

Fig. 5 : Le tableau sur le mur derrière Ffi 

pleine de détresse de Liam : «Show it to me! I want to see it! I want to see what he looks like! This isn't me! Look at what you're doing to me! » Le lapsus de Liam (et on sait l'importance des lapsus dans cet épisode), qui utilise « he » plutôt que «it » pour évoquer le préservatif ou le sexe de Jonas, laisse entendre qu'à sa jalousie pathologique s'ajoute un désir homosexuel inavoué : c'est lui qui désire «Mister Cool » et qui reconnaît son désir reflété sur le visage de sa compagne. L'épisode est d'ailleurs placé sous le signe du narcissisme de Liam, puisqu'il s'ouvre et se clôt sur l'image du reflet du jeune homme (cf. Fig. 6) :

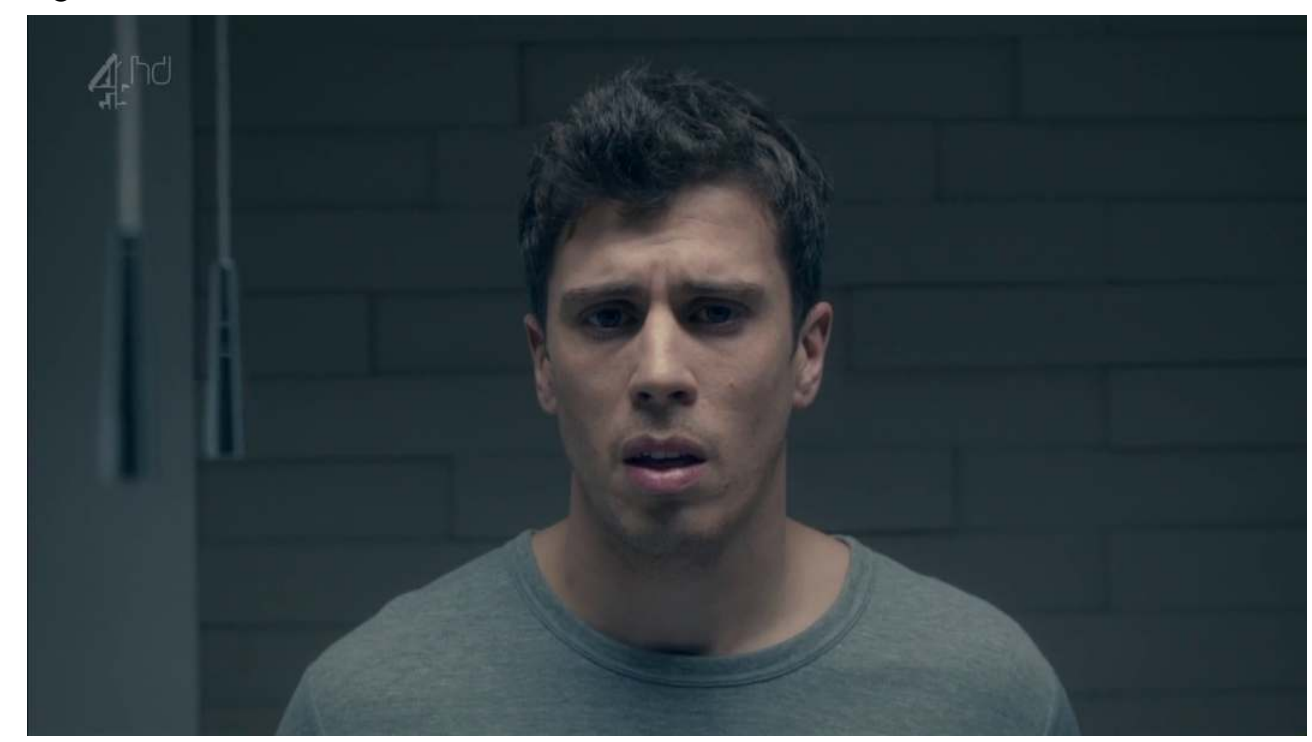

Fig. 6 : L'image de Liam

Comme chez David Cronenberg (on pense à Dead Ringers de 1988 en particulier), la technologie et la femme servent de médiateurs aux désirs tabous du sujet masculin, corrélation permise par l'analogie que nos cultures établissent, selon Mary Ann Doane ${ }^{34}$, entre la technologie et le féminin. Les rediffusions révéleraient bien une vérité, mais pas forcément (ou du moins pas seulement) celle que Liam considère; on en revient aux limites de l'auto-analyse.

La relation qu'entretient Liam avec les rediffusions semble d'ailleurs largement partagée au niveau diégétique. "You know half the organic memories you have are junk. Just not trustworthy », dit Colleen (qui travaille dans le développement du Grain) à Halem, avec une certaine condescendance. Sa remarque trahit une confusion entre memory et memorabilia, suggérée par l'emploi du mot «junk». L'appréciation du Grain par les personnages repose donc sur une double croyance: en la vérité infaillible de l'image photographique, mais aussi en l'idée que l'ontologie du numérique est finalement la même que celle du photographique. Or, le numérique implique bel et bien un changement de régime ontologique. Comme le remarque D.N. Rodowick : 
Where analog media record traces of events, as Binckley put it, digital media produce tokens of numbers [...] [digital imagery] has loosed its anchors from both substance and indexicality. And it is not simply that visuality has been given a new mobility wherein any pixel in the electronic image can be moved or its value changed at will ${ }^{35}$. technologie qui la précède; l'exemple le plus criant est sans doute l'effet sonore de rembobinage ou d'avance rapide qui imite le son d'une cassette audio ${ }^{36}$. Cette capacité à se faire passer pour du film est, d'ailleurs, comme Donna Kornhaber le note dans son étude de Her, une des caractéristiques de l'audiovisuel numérique ${ }^{37}$. On comprend alors pourquoi les rediffusions ne se distinguent pas radicalement des enregistrements effectués par une caméra super-8 ou vidéo, et ne permettent finalement rien de plus qu'une démultiplication de leurs possibles: facilité de production, de stockage, de transfert, etc.

Cette double croyance implique également un double assujettissement au discours du pouvoir. Colleen renchérit en ajoutant: «With half the population, you can implant false memories just by asking leading questions in therapy. You can make people remember getting lost in shopping malls they never visited, getting bothered by pedophile babysitters they never had. » Et pourtant le sujet n'est pas à l'abri d'une modification de ses rediffusions, ni d'un ajout dans sa base de données ${ }^{38}$. Il suffit de penser au postulat de «The Minority Report» de Philip K. Dick et de son adaptation (Steven Spielberg, 2002). C'est donc dans un aveuglement consternant que Colleen, cas type de l'employée relayant le discours de l'entreprise ou de l'institution, véhicule un discours marketing reposant sur la conviction qu'il est impossible de manipuler des images (photographiques ou numériques, puisque finalement c'est la même chose) et, ce faisant, renforce potentiellement la capacité de contrôle du pouvoir ${ }^{39}$. Car même si ce n'est pas le cœur de "The Entire History of You », qui est centré sur la sphère intime, le traitement de l'espace laisse entendre que cette société, dans laquelle la frontière entre privé et public est mince, sert un régime de contrôle. L'architecture toute entière du monde diégétique est caractérisée par la présence de grandes fenêtres, signifiant à la fois que tout y est ouvert et visible, y compris l'espace des domiciles, mais suggérant aussi que le sujet est toujours perçu par le prisme de surfaces réfléchissantes - en effet, le miroir du taxi est également un écran (cf. Fig. 7). 


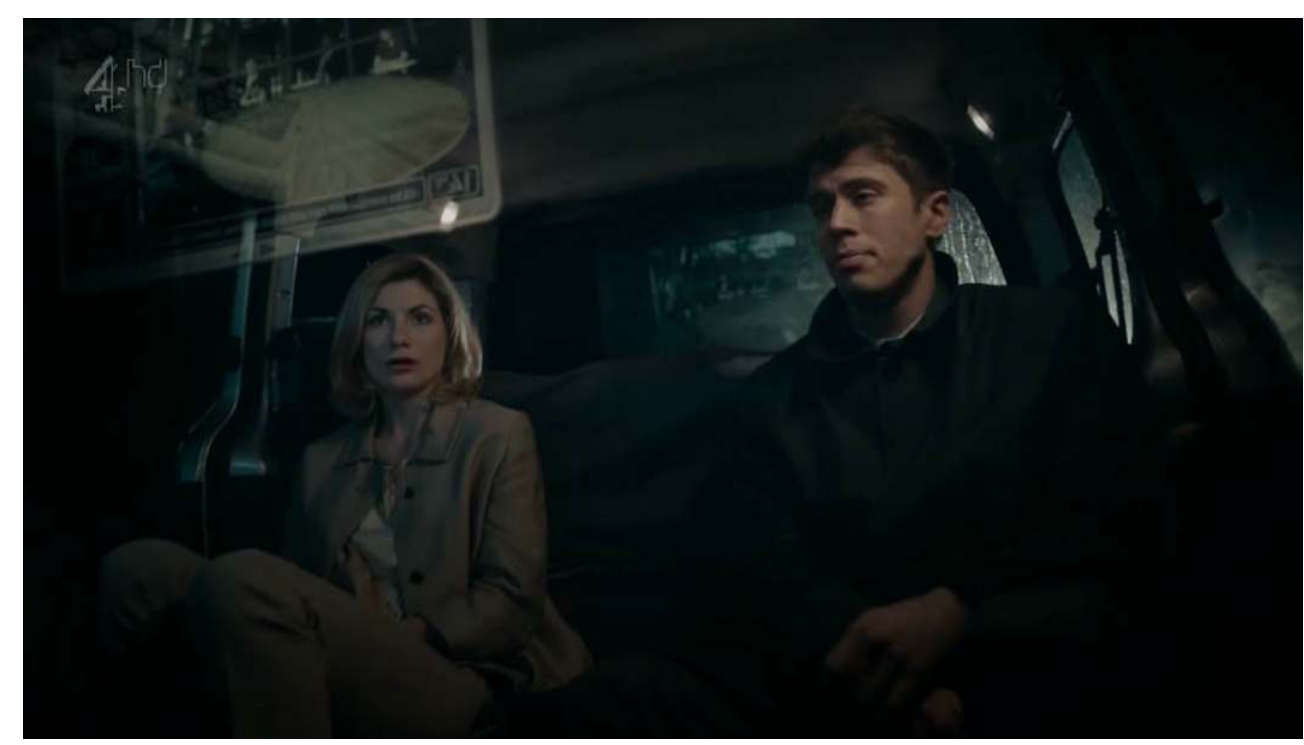

Fig. 7 : Le miroir du taxi comme écran

$\mathrm{du}$ monde qui freine le devenir posthumain. Le sujet posthumain est prisonnier de l'image, des écrans, des reflets, et l'implant n'est finalement que l'allégorie du fait que notre perception est bel et bien façonnée par les caméras et les écrans ${ }^{41}$. Si la distinction entre images actuelles et virtuelles est acquise, et est maintenue jusqu'à l'avant-dernière scène grâce aux contrastes entre les couleurs froides et argentées du présent et les tons plus lumineux et chaleureux du passé, la distinction est moins claire dans la scène finale suite à la rupture entre Liam et Ffi. Liam fait ici un emploi très bazinien des rediffusions qui relève du "complexe de la momie", à savoir le désir de préservation et de commémoration du passé auquel, selon Bazin, l'image a toujours eu vocation depuis l'Antiquité $^{42}$. Le montage ressemble à un champ/contrechamp monté en raccord-regard avec le personnage observé (marqué par les yeux lumineux) et le personnage observant. Qu'il s'agisse du passé ou du présent, c'est son reflet et celui de Ffi auxquels il fait face. Il y a donc incertitude concernant le statut de l'image et du montage - du moins jusqu'à ce que la caméra opère un panoramique vers le bas et que le plan se révèle comme subjectif, justement au moment même où Liam décide d'extraire la prothèse mnésique, c'est-à-dire au moment où le regard cède la place au toucher. Ce segment est un cas d'école de l'« image-cristal » deleuzienne, image qui opère une confusion entre l'image actuelle et son image virtuelle, et dont la qualité est de faire l'expérience directe du temps et donc de la subjectivité ${ }^{43}$. Mais au final, ce changement de régime sensible - de l'image-mouvement à l'image-temps - ne fait que résumer l'histoire du cinéma selon Deleuze. Autrement dit, si cette image-cristal marque un tournant dramatique et sensible dans l'épisode, elle n'en demeure pas moins une modalité héritée du récit audiovisuel analogique, et donc d'un paradigme passé, preuve, s'il en faut, qu'au niveau esthétique l'on est encore bien loin du post-cinéma.

30 Malgré la référence à Gibson, et à l'exception de "San Junipero », « USS Callister » et «Hang the DJ », la série Black Mirror est farouchement dystopique et bien plus prudente 
que les œuvres qui l'ont influencée quant à l'attrait du posthumain ou du transhumanisme ${ }^{44}$. La série anthologie, dont la sérialité réside principalement dans la multiplication de possibilités, et donc dans la manière dont les épisodes se font écho (d'où la centralité du miroir comme motif), ne fait pourtant pas preuve de technophobie primaire mais, au contraire, tout à fait réfléchie. Il ne s'agit pas de diaboliser la technologie mais d'insister sur les limites de son emploi, limites qui sont principalement dues à une répugnance à pleinement se tourner vers l'avenir. Dans les épisodes S01E03, S03E01, S03E02, S03E05, S04E02, S04E03 et S04E06 de Black Mirror, les implants ne font que perpétuer des modèles passés («Arkangel » va jusqu'à recycler la technologie de «The Entire History of You ») et exacerber certaines modalités de cognition et du sensible au détriment d'autres, sans pour autant en offrir de nouvelles ; quant aux potentialités, leur emploi demeure principalement ludique, obsessionnel, narcissique, tyrannique et ne laisse aucunement présager de la révolution numérique collective souhaitée par Jenkins. Ce discours sur le devenir posthumain est également un discours métafictionnel sur la spectature et les médias, et donc sur l'identité humaine et médiatique, et sur la création de soi et d'histoires, comme le titre de l'épisode l'indique. Alors même que la série souligne les tendances souvent néfastes du revisionnage, et donc de notre statut contemporain de "revoyants », elle nous invite à la revoir et, de manière plus générale, à penser le revisionnage comme exercice critique. Mais elle semble aussi regretter ses propres limites : à savoir qu'en tant que série grand public diffusée à la télévision et maintenant sur Netflix, elle reste prisonnière de l'héritage d'un format, d'une narration classique et d'un mode de diffusion qui n'exploitent pas toutes les potentialités du numérique et de la culture de la convergence. D'une certaine manière, Black Mirror semble désolée que sa forme ne soit finalement pas plus en adéquation avec son propos, peutêtre parce que, comme Liam lors de son entretien d'embauche, son showrunner demeure sous le contrôle d'une industrie qui exige la rentabilité sous peine de chômage... et d'écran noir définitif.

\section{NOTES}

1. Thierry Hoquet, «Cyborg, Mutant, Robot, etc. : Essai de typologie des presque-humains », in Post Humains. Frontières, évolutions, hybridités, éd. Elaine Després et Hélène Machinal, Rennes, PUR, 2014, p. 99-118.

2. Charlie Brooker, "The Dark side of our gadget addiction ", The Guardian, $1^{\mathrm{er}}$ décembre 2011, https://www.theguardian.com/technology/2011/dec/01/charlie-brooker-dark-side-gadgetaddiction-black-mirror.

3. William Gibson, Neuromancer, New York, Ace Books, 1984.

4. Sébastien Lefait, «"It's not a technological problem we have, it's a human one" : Black Mirror, ou la dystopie intégrée », Otrante, $\mathrm{n}^{\circ} 42,2017$, p. 127-144.

5. Donna Kornhaber, «From Posthuman to Postcinema: Crises of Subjecthood and Representation in Her », Cinema Journal, n56.4, 2017, p. 3-25.

6. Henry Jenkins, Convergence Culture: Where Old and New Media Collide, Updated and with a New Afterword, New York et Londres, New York University Press, 2008 [2006]. 
7. Scott Bukatman, Terminal Identity: The Virtual Subject in Post-Modern Science Fiction, Durham, Duke University Press, 1993. Mehdi Achouche, L'Utopisme technologique dans la science-fiction hollywoodienne, 1982-2013 : transhumanisme, posthumanité et le rêve de "l'homme-machine ", Thèse de doctorat, Université Stendhal Grenoble 3, 2011.

8. Lefait, "'It's not a technological problem we have, it's a human one" : Black Mirror, ou la dystopie intégrée », p. 128.

9. Voir l'article d'Hélène Machinal dans ce numéro de TV/Series.

10. Pour une application du concept inventé par Steve Mann à des fictions audiovisuelles anglophones, voir Sébastien Lefait, Surveillance on Screen: Monitoring Contemporary Films and Television Programs, Lanham (Maryland), Scarecrow Press, 2013.

11. Acte $1: 2: 50-3: 23 ; 4: 50-5: 02$. Acte $2: 14: 02-15: 55 ; 19: 35-20: 10$. Acte $3: 22: 20-30: 24$. Acte 4 : $34: 35-36: 23 ; 38: 03-38: 40 ; 42: 34-43: 19 ; 43: 30-46: 21$.

12. On retrouve les mêmes inquiétudes dans les épisodes S03E01, S03E02, S03E05 et S04E06. Les implants des épisodes « Arkangel » (S04E02) et « Crocodile » (S04E03) et l'interface de notation de " Nosedive » permettent de savoir à tout moment où se trouve un sujet donné et/ou de fouiller dans ses archives, tandis que les implants de «Playtest » et de "Men Against Fire » altèrent la perception du réel du joueur ou des soldats et même leur imaginaire; l'implant de l'épisode S03E05 atténue également les autres sens (odorat, goût).

13. C'est d'ailleurs cette idée qu'approfondit « Nosedive ».

14. La camionneuse que rencontre Lacie Pound tient le même rôle dans « Nosedive ».

15. La première histoire de l'épisode «Black Museum » propose alors un contre-point puisque c'est l'overdose sensorielle du toucher en particulier qui conduit à l'aliénation de Dawson.

16. Il en est de même dans " Nosedive » dans lequel les personnages jouent en étant conscients d'être constamment notés ; cette conscience détermine d'ailleurs le jeu des acteurs qui surjouent - voire qui « sur-sourient » derrière leurs maquillages, coiffures et costumes excessifs - de façon grotesque.

17. « Nosedive » s'intéresse plus au jeu.

18. Voir Laurent Jullier, Cinéma et cognition, Paris, L'Harmattan, 2000, p. 94.

19. L'implant nommé MASS dans « Men Against Fire » reprend ce principe.

20. Jean-Louis Baudry, L'Effet cinéma, Paris, Albatros, 1978. Christian Metz, Le Signifiant imaginaire: Psychanalyse et cinéma, Paris, Christian Bourgeois, 2002 [1977]. Laura Mulvey, «Visual Pleasure and Narrative Cinema » (1975), Visual and Other Pleasures, Bloomington, Indiana University Press, 1989, p. 14-26.

21. David Bordwell, Narration in the Fiction Film, Madison, University of Wisconsin Press, 1985. Murray Smith, Engaging Characters: Fiction, Emotion, and the Cinema, Oxford et New York, Oxford University Press, 1995. S. Tan (ed.), Emotions and the Structure of Narrative Film: Film as an Emotion Machine, Mahwah, Lawrence Erlbaum Associates, 1996.

22. Dans «Playtest ", le concepteur de jeu vidéo (du moins celui imaginé par le protagoniste) propose un amalgame de théorie psychanalytique et de cognitivisme écologique pour expliquer le plaisir que procurent les jeux de type survival horror : «But mostly because you are still alive. You have faced your greatest fears in a safe environment. It is a release of fear. It liberates you. » 23. Il en est de même dans l'épisode « Men Against Fire » où elle signifie l'aveuglement du sujet à l'humanité de ceux qu'il perçoit comme des cafards (Roaches).

24. Il en est de même du téléphone dans « Nosedive ».

25. Lefait, " "It's not a technological problem we have, it's a human one" : Black Mirror, ou la dystopie intégrée », p. 139.

26. Il en est de même dans « Nosedive ».

27. Jenkins, op. cit., p. 219.

28. Il me semble que c'est aussi le cas dans un épisode «Crocodile » qui fait au bout du compte la démonstration du caractère implacable de la sousveillance. 
29. Rosalind Krauss, Le Photographique: Pour une théorie des écarts, Paris, Éditions Macula, 1990, p. 13-14.

30. André Bazin, «Ontologie de l'image photographique " (1945), Qu'est-ce que le cinéma? Paris, Les Éditions du Cerf, 2002, p. 13.

31. Susan Sontag, On Photography, New York et Londres, Penguin, 1977, p. 154.

32. Roland Barthes, La Chambre claire : note sur la photographie, Paris, Éditions de l'Étoile, Gallimard, Le Seuil, 1980, p. 120.

33. "Playtest» fait d'ailleurs du jeu vidéo une ramification nouvelle du "mythe du cinéma total " théorisé par Bazin. André Bazin, «Le Mythe du cinéma total » (1946), Qu'est-ce que le cinéma ? Paris, Les Éditions du Cerf, 2002, p. 19-24.

34. Mary Ann Doane, "Technophilia: Technology, Representation, and the Feminine ", in Body/ Politic: Women and the Discourses of Science, ed. Mary Jacobus, Evelyn Fox Keller et Sally Shuttleworth, New York, Routledge, 1990, p. 163.

35. D.N. Rodowick, The Virtual Life of Film, Cambridge, Harvard University Press, 2007, p. 9-10.

36. Il en est de même dans "Men Against Fire " où le MASS réduit les sens humains à une perception audiovisuelle.

37. Kornhaber, op. cit., p. 22.

38. Cette possibilité est justement explorée dans « Men Against Fire ».

39. Ce danger mis en avant dans les épisodes « Arkangel » et «Crocodile » où la technologie est quasi-identique.

40. Les épisodes «Playtest » et « Men Against Fire » usent aussi de caméras de surveillance.

41. Lefait, " "It's not a technological problem we have, it's a human one" : Black Mirror, ou la dystopie intégrée », p. 138-39.

42. Bazin, « Ontologie de l'image photographique », op. cit., p. 9.

43. Gilles Deleuze, Cinéma 2 : L'Image-temps, Paris, Les Éditions de Minuit, 1985, p. 93-94, p. 110.

44. Lefait, " "It's not a technological problem we have, it's a human one" : Black Mirror, ou la dystopie intégrée », p. 143.

\section{RÉSUMÉS}

Cet article porte sur la série anthologique Black Mirror (2011-), créée par Charlie Brooker et d'abord diffusée par Channel 4, puis par Netflix à partir de la saison 3. Il s'intéresse tout particulièrement à l'épisode «The Entire History of You » (S01E03), avec des incursions dans six épisodes des saisons 3 et 4 - « Nosedive » (S03E01) «Playtest» (S03E02), « Men Against Fire » (S03E05), «Arkangel », (S04E02) «Crocodile» (S04E03) et «Black Museum» (S04E06). On verra comment, sous couvert d'un questionnement éthique assez convenu sur le devenir-cyborg (ou l'humain augmenté), l'épisode S01E03, comme la série de façon générale, élabore un discours métafictionnel en s'interrogeant sur le devenir de la mémoire humaine à l'ère du numérique, de la surveillance et de la culture de la convergence. La mise en abyme de la consommation d'images de soi et d'autrui permet d'interroger les modes de diffusion propre à la série. L'épisode suggère que, plutôt que de révolution technologique, c'est encore et toujours le statut du photographique qui pose problème au sujet humain, du fait de sa reproductibilité et de la perte de l'aura auquel il donne lieu. Ce faisant, la série insiste sur la continuité ontologique entre anciens et nouveaux médias qu'elle pense comme des « êtres en cours ». 
Posthuman memory, being and memory, memory and the senses - it is not so much these themes that account for the novelty of Black Mirror, a series that clearly assumes its cinematic, literary and televisual heritage. What makes the series so compelling is that the exploration of technology goes far beyond social allegory; Black Mirror proposes metafictional reflections on how audiovisual fictions like the series itself are created, circulated and consumed in the contemporary mediascape - granted, to various degrees depending on the episodes, hence the particular focus on "The Entire History of You". This chapter opens with a discussion of the utopian and dystopian potential of the various implants - or rather of their uses - in a series that is known for its technophobia. It is followed by a study of the thematization of spectatorship, which is indebted to both the psychoanalytical model of the 1970s and the cognitive model of the late 1980s-1990s; though the spectator-actors depicted in the diegeses demonstrate a certain know-how, their inability to exploit the analytical and political potential of the new media ultimately voices the series' worries about surveillance/sousveillance societies and doubts concerning the convergence culture it belongs to. This failure, I argue in the last section, may partially be explained by the fact that the implants have ultimately been modeled on the technology of the past; more precisely, digital is made into a mere continuation of the photographic that expands the Bazinian characteristics of the latter without radically tapping into the specific ontology of the digital image. In so doing, Black Mirror demonstrates the high degree of self-consciousness characteristic of metafiction, proposing a complex discourse on its form and its culture, all the while apologizing for the fact that its form remains chained to past formats and media, and thus for the fact that it is not more in tune with its own metadiscourse.

\section{INDEX}

Keywords : Black Mirror, implant, medium, memory, metafiction, posthuman, spectatorship, subjectivity, technology

Mots-clés : Black Mirror, implant, média, mémoire, métafiction, posthumain, spectature, subjectivité, technologie

\section{AUTEUR}

\section{DAVID ROCHE}

David Roche est professeur d'études cinématographiques au DEMA à l'Université Toulouse Jean Jaurès et Président de la SERCIA (www.sercia.net). Il est l'auteur de Quentin Tarantino: Poetics and Politics of Cinematic Metafiction (University Press of Mississippi, 2018) et de Making and Remaking Horror in the 1970s and 2000s (2014), et a co-dirigé plusieurs collectifs, dont Steven Spielberg, Hollywood Wunderkind \& Humanist (2018), Bande dessinée et adaptation (2015) et De l'intime dans le cinéma anglophone (2015). Il travaille actuellement sur la métafiction dans le cinéma et les séries. David Roche is Professor of Film Studies at the Université Toulouse Jean Jaurès, France, and President of SERCIA (www.sercia.net). He is the author of Quentin Tarantino (University Press of Mississippi, 2018) and Making and Remaking Horror in the 1970s and 2000s (2014) and the editor of Steven Spielberg, Hollywood Humanist \& Wunderkind (PULM, 2018) and Comics and Adaptation (2018). His work on American, British and Canadian art and horror cinema has appeared in Adaptation, CinémAction, Horror Studies, Post-Script and Transatlantica. He is currently working on metafiction in film and series. 